\title{
Primary Neural Degeneration in Noise-Exposed Human Cochleas: Correlations with Outer Hair Cell Loss and Word-Discrimination Scores
}

\author{
Pei-Zhe Wu, ${ }^{1,2,3}$ Jennifer T. O’Malley, ${ }^{1}$ Victor de Gruttola, ${ }^{4}$ and ${ }^{\circledR}$ M. Charles Liberman ${ }^{1,2}$ \\ ${ }^{1}$ Eaton-Peabody Laboratories, Massachusetts Eye and Ear, Boston, Massachusetts 02114, ${ }^{2}$ Department of Otolaryngology, Harvard Medical School, \\ Boston, Massachusetts $02115,{ }^{3}$ Department of Otorhinolaryngology Head and Neck Surgery, The First Affiliated Hospital, Sun Yat-sen University, \\ Guangzhou 510080, China, and ${ }^{4}$ Department of Biostatistics, Harvard T.H. Chan School of Public Health, Boston, Massachusetts 02115
}

Animal studies suggest that cochlear nerve degeneration precedes sensory cell degeneration in both noise-induced hearing loss (NIHL) and age-related hearing loss (ARHL), producing a hearing impairment that is not reflected in audiometric thresholds. Here, we investigated the histopathology of human ARHL and NIHL by comparing loss of auditory nerve fibers (ANFs), cochlear hair cells and the stria vascularis in a group of 52 cases with noise-exposure history against an age-matched control group. Although strial atrophy increased with age, there was no effect of noise history. Outer hair cell (OHC) loss also increased with age throughout the cochlea but was unaffected by noise history in the low-frequency region $(<2 \mathrm{kHz})$, while greatly exacerbated at high frequencies $(\geq 2 \mathrm{kHz}$ ). Inner hair cell (IHC) loss was primarily seen at high frequencies but was unaffected by noise at either low or high frequencies. ANF loss was substantial at all cochlear frequencies and was exacerbated by noise throughout. According to a multivariable regression model, this loss of neural channels contributes to poor word discrimination among those with similar audiometric threshold losses. The histopathological patterns observed also suggest that, whereas the low-frequency $\mathrm{OHC}$ loss may be an unavoidable consequence of aging, the high-frequency loss, which produces the classic down-sloping audiogram of ARHL, may be partially because of avoidable ear abuse, even among those without a documented history of acoustic overexposure.

Key words: hidden hearing loss; human temporal bone; inner ear; noise exposure; word recognition

Significance Statement

As regenerative therapeutics in sensorineural hearing loss enter clinical trials, it becomes critical to infer which cochlear pathologies are present in addition to hair cell loss. Here, by analyzing human autopsy material, we show that acoustic injury accelerates age-related primary neural degeneration, but not strial degeneration, neither of which can be inferred from audiometric thresholds. It exacerbates outer hair cell $(\mathrm{OHC})$ loss only in the high-frequency half of the cochlea, suggesting that the apical loss is age-related, whereas the basal loss is partially noise induced, and therefore avoidable. Statistical analysis suggests that neural loss helps explain differences in word-recognition ability among individuals with similar audiometric thresholds. The surprising correlation between neural loss and OHC loss in the cochlea's speech region also implicates neural loss in the well-known decline in word scores as thresholds deteriorate with age.

Received Dec. 30, 2020; revised Mar. 23, 2021; accepted Mar. 27, 2021.

Author contributions: P.Z.W., J.T.O., and M.C.L. designed research; P.Z.W. and J.T.0. performed research; P. Z.W. contributed unpublished reagents/analytic tools; P.Z.W. and V.d.G. analyzed data; P.Z.W. and M.C.L. wrote the paper.

This work was supported by the National Institute on Deafness and Other Communication Disorders Grant P50 DC 015857 and the Lauer Tinnitus Center at the Massachusetts Eye and Ear. We thank the assistance of all the members of the Otopathology Laboratory at Massachusetts Eye and Ear.

The authors declare no competing financial interests.

Correspondence should be addressed to Pei-Zhe Wu at Peizhe_Wu@meei.harvard.edu.

https://doi.org/10.1523/JNEUROSCI.3238-20.2021

Copyright $\odot 2021$ the authors

\section{Introduction}

In animal models, acoustic overexposures can cause widespread destruction of the synapses between auditory nerve fibers (ANFs), the primary sensory neurons of the auditory pathway, and their peripheral targets, the cochlea's inner hair cells (IHCs; Kujawa and Liberman, 2009; Furman et al., 2013). This immediate synaptopathy is followed, more slowly, by a loss of the peripheral axons of these bipolar sensory neurons, and ultimately by degeneration of their cell bodies in the spiral ganglion (Liberman and Kiang, 1978; Kujawa and Liberman, 2009). This partial de-afferentation of the sensory cells does not affect the threshold audiogram until $>80 \%$ of the fibers have been silenced (Schuknecht and Woellner, 1955; Lobarinas et al., 2013). 
However, differences in the severity of this primary neural degeneration may help explain why people with similar threshold audiograms can have different speech-recognition abilities, especially in noisy environments, a common complaint among those with sensorineural hearing loss. ANF loss reduces information channels to the central nervous system; and, in animals, this can compromise discrimination of complex stimuli without affecting the ability to detect tones in quiet (Chambers et al., 2016), which is the task on which the audiogram is based.

Determining whether primary neural degeneration is a major component of acoustic injury in humans is important to the public health, given that workplace noise guidelines are based on the assumption that permanent threshold shifts are the only relevant metric of permanent cochlear damage (Arenas and Suter, 2014). Electrophysiological studies comparing auditory evoked potentials in people with similar thresholds but differing noise histories have come to different conclusions about the prevalence of noise-induced cochlear neuropathy (Liberman et al., 2016; Prendergast et al., 2017).

Acoustic overexposure can also damage the stria vascularis (Wang et al., 2002), the "cochlear battery" that generates the electrical gradient driving mechanoelectric transduction. Since strial atrophy and hair cell death both elevate thresholds, the two types of damage are not clearly distinguishable from audiometric data (Wu et al., 2020a). Inferring the relative damage to different cochlear structures from noninvasive measures, and from hearing-loss etiology, is increasingly important, as therapeutics for hair cell and/or neural regeneration are transitioning from preclinical models to clinical trials (Kujawa and Liberman, 2019). Since the inner ear cannot be biopsied, the only unambiguous way to assess tissue damage in the human cochlea is by examination of postmortem material. Here, we quantify histopathology in cochlear specimens from the archival collection at the Massachusetts Eye and Ear (Merchant et al., 2008), comparing those with noise-exposure history (typically occupational), to "normal-aging" controls.

Our results suggest that degeneration of IHCs and the stria vascularis are unaffected by noise history, while outer hair cell (OHC) loss is exacerbated in the basal (high-frequency) cochlea, and ANF loss is exacerbated throughout. Statistical modeling of histopathological metrics as predictors of audiometric outcomes suggested that ANF loss contributes to poor speech discrimination by partially de-afferenting surviving IHCs in both low-frequency and high-frequency cochlear regions. The histopathological patterns also suggest that hair cell loss in the low-frequency cochlea may be an unavoidable consequence of aging, whereas the $\mathrm{OHC}$ loss in the high-frequency regions, which creates the classic "down-sloping" audiogram of aging, is at least partially because of a lifetime of ear abuse, even among those without exceptionally noisy jobs.

\section{Materials and Methods}

\section{Subjects and groups}

Archival slide sets of serially sectioned, celloidin-embedded temporal bones from the Massachusetts Eye and Ear collection were analyzed. A survey of the otologic histories yielded 52 ears containing key words: "acoustic trauma" or "noise" and met the following inclusion criteria: (1) adequate preservation for reliable microscopic analysis; (2) no genetically determined or developmental defects; (3) no history of infections, neoplastic growth, vascular disorders, or bone malformation in the ear; (4) no history of otologic surgery; (5) no history of vertigo, dizziness, or sudden sensorineural hearing loss; (6) no autoimmune disease; and (7) no history of exposure to ototoxic drugs. The selected individuals ranged in age from 43 to 104 years and included 47 males and 5 females (Table
Table 1. Sex, ages, ears analyzed, occupational exposures, and causes of death for all the cases classified as "noise history" in the present study

\begin{tabular}{|c|c|c|c|c|}
\hline Sex & Age & Side & Exposure & Cause of death \\
\hline M & 43 & L & Military service & Unknown \\
\hline M & 52 & $R, L$ & Ship engine room & Pulmonary emboli \\
\hline M & 58 & $\mathrm{R}, \mathrm{L}$ & Ship builder & Chondrosarcoma \\
\hline M & 59 & $\mathrm{R}, \mathrm{L}$ & Pipeline worker & Medullary carcinoma \\
\hline M & 60 & L & Truck driver & Amyotrophic lateral sclerosis \\
\hline M & 61 & $R, L$ & Military service, coppersmith & Gastrointestinal bleeding \\
\hline M & 62 & $R, L$ & Foundry worker & Meningotheliomatous \\
\hline M & 63 & $\mathrm{R}$ & Military service & Cardiac arrest \\
\hline M & 66 & $\mathrm{~L}$ & Subway conductor & Stroke \\
\hline M & 68 & $R, L$ & Military service: jet engines & Lung cancer \\
\hline M & 69 & $\mathrm{R}, \mathrm{L}$ & Noisy machine & Lung cancer \\
\hline M & 70 & $\mathrm{R}$ & Carpenter & Carotid artery rupture \\
\hline M & 71 & $\mathrm{R}, \mathrm{L}$ & Steel worker & Myocardial infarction \\
\hline M & 72 & $\mathrm{R}$ & Construction worker & Necrotizing bronchopneumonia \\
\hline M & 75 & $\mathrm{R}$ & Welder, riveter & Lung cancer \\
\hline M & 76 & $R, L$ & Automobile factory worker & Pulmonary edema, myocardial infarction \\
\hline M & 76 & $\mathrm{~L}$ & Laundry worker & Congestive heart failure, renal failure \\
\hline M & 76 & $R, L$ & Factory worker & Unknown \\
\hline M & 77 & $\mathrm{R}$ & Hunter & Unknown \\
\hline M & 77 & $R, L$ & Newspaper pressman & Myocardial infarction \\
\hline M & 78 & $\mathrm{R}$ & Military service & Unknown \\
\hline $\mathrm{F}$ & 81 & $\mathrm{R}$ & Musician & Myocardial infarction \\
\hline M & 81 & $\mathrm{R}, \mathrm{L}$ & Machinery mover & Myocardial infarction \\
\hline M & 81 & $\mathrm{R}, \mathrm{L}$ & Factory worker & Septicemia \\
\hline M & 82 & L & Riveter & Gastrointestinal bleeding \\
\hline M & 83 & R & Military service & Stroke, pulmonary emboli \\
\hline M & 84 & $R, L$ & Military service & Stroke \\
\hline M & 87 & $\mathrm{R}$ & Prize fighter & Lung cancer \\
\hline $\mathrm{F}$ & 89 & L & Factory seamstress & Lung cancer \\
\hline $\mathrm{F}$ & 90 & $R, L$ & Factory worker & Progressive supranuclear palsy \\
\hline M & 94 & $\mathrm{R}, \mathrm{L}$ & Self-exposed researcher & Unknown \\
\hline M & 95 & $\mathrm{R}, \mathrm{L}$ & Self-exposed researcher & Unknown \\
\hline$F$ & 96 & $\mathrm{R}$ & Factory seamstress & Stroke, congestive heart failure \\
\hline M & 104 & $R, L$ & Ship builder & Unknown \\
\hline
\end{tabular}

For cases with left and right ears, each side is treated as an individual case.

$1)$. We identified an age-matched group without evidence of noise exposure history $(n=51)$, ranging in age from 41 to 93 years, and including 20 males and 31 females, who also met the criteria stated above. The agematched control group was supplemented by 17 younger cases, from 0 to 39 years, to assess the age-related changes in the normal group.

Of these 120 cases, 66 had word-recognition scores (expressed as a percentage of monosyllabic, phonetically balanced words correctly identified when presented in quiet at an audible level) and complete histopathological measurements, and, in 60 of them, the audiological assessments were within six years of death; six cases with word scores taken from 8 to 18 years before death were also included. The longer the test-death interval, the greater the risk of underestimating hearing impairment, since in normal aging or after noise damage, hearing only deteriorates with time (Gordon-Salant, 2005). Many of the cases analyzed here also contributed to a prior study of normal aging in the human cochlea (Wu et al., 2020a). All procedures and protocols for the study of human tissues included informed consent and were approved by the Institutional Review Board of the Massachusetts Eye and Ear. The custom computer code as well as the datasets generated and/or analyzed for the current study are available from the corresponding author on reasonable request.

\section{Histologic analysis}

For all histologic analysis, observers were blinded to otologic history and audiometric data. For each case, the set of 40-50 archival slides contains $\sim 100$ cuts through the spiraling cochlear duct. The cochlear spiral in each case was graphically reconstructed (Merchant and Nadol, 2010) to allow the percent distance along the spiral of each section through the 
cochlear duct to be computed and converted into frequency $(\mathrm{kHz})$ using a cochlear map for human (Greenwood, 1990), as modified to produce apical-most and basal-most best frequencies of 0.1 and $20 \mathrm{kHz}$, respectively.

\section{Tissue processing}

When originally archived, each temporal bone was aldehyde fixed, decalcified, embedded in celloidin, and serially sectioned at $20 \mu \mathrm{m}$ in the horizontal plane. Every 10th section was stained with hematoxylin/eosin and slide-mounted (Merchant and Nadol, 2010), while intervening (unstained) sections were stored in $80 \%$ alcohol. For the present study, selected unstained sections were retrieved, de-celloidinized (O'Malley et al., 2009) and stained with a fluorescent membrane dye (CellMask Orange, ThermoFisher \#C10045) at 1:1000 for $5 \mathrm{~min}$ to label myelin sheaths. The sections were then coverslipped with Vectashield.

\section{Hair cell counts}

Fractional survival of IHC and OHC was quantified in all archival sections from each case using a $100 \times$ oil-immersion objective $(\mathrm{NA}=1.3$ ) and differential interference optics on a Nikon E800 microscope. In each section through the cochlear duct, the number of remaining cells in each hair cell row was counted, using cuticular plates and hair bundles as the main criterion for identifying hair cells. The number of missing cells was estimated from (1) the size of the gaps between surviving cells; (2) the number of nearby supporting cells; and (3) mean hair cell counts from young ears, as described in more detail elsewhere (Wu et al., 2020b). Dividing the two values yields fractional survival, which needs no further normalization. Hair bundles and cuticular plates were the main criterion for surviving hair cells, rather than cell nuclei. The hair cell analysis in each case is based on observations at $\sim 100$ positions along the cochlear spiral.

\section{Peripheral axon counts}

Neural degeneration was assessed by counting peripheral axons of ANFs, rather than their cell bodies, because cell bodies can survive for years after peripheral axon loss (Chen et al., 2006; Kujawa and Liberman, 2009; Liu et al., 2015). ANFs were quantified at five locations along the cochlear spiral, one at the tangential section through each half turn, where the axons are cut in cross section and can be precisely counted (Wu et al., 2018). Previously unstained sections were selected from the archive for each half turn, seeking the section $200 \mu \mathrm{m}$ proximal to the one containing IHC tangent. After staining, confocal $z$-stacks were acquired with $0.33-\mu \mathrm{m}$ z-spacing on a Leica SP8 using a $63 \times$ glycerol objective $(\mathrm{NA}=1.3)$. Image stacks spanning the top $5 \mu \mathrm{m}$ of the section were acquired. The brightest focal plane was selected, and axons were counted within a $250-\mu \mathrm{m}$ mask. Raw data were normalized to average counts from the appropriate cochlear region in six young normal cases, aged 0-5.4 years (Wu et al., 2018).

\section{Strial analysis}

Strial atrophy was quantified in the archival slide sets by measuring the cross-sectional area of the stria at 13 cochlear locations, equally spaced at roughly $1 / 8$ of each cochlear turn, avoiding regions where the stria is cut tangentially. At each locus, healthy strial tissue was outlined and measured using computer-aided anatomy software (Neurolucida). The sections were displayed through a video camera connected to a Nikon E600 using a $20 \times$ objective $(\mathrm{NA}=0.75)$. One focal plane, near the middle of the section, was chosen for tracing. Raw data were normalized to average data from the appropriate cochlear region in young normals $(n=5)$, aged $0-5.4$ years.

\section{Statistical modeling}

To select the functionally most important histologic structures and to fit a model, linear regression was performed via the least absolute shrinkage and selection operator (LASSO) method using the glmnet package in R 3.6.1. Before fitting in the LASSO linear regression, the data were preprocessed: (1) histopathological metrics were averaged separately across low $(0.25,0.5$, and $1.0 \mathrm{kHz})$ and high $(2.0,4.0$, and $8.0 \mathrm{kHz})$ audiometric frequencies and were treated as continuous variables; and (2) all variables (i.e., histopathological measurements, hearing levels and word scores) were normalized ( $z$ score). The best model was selected via 10 fold cross-validation. The statistical significance of between-group differences, when the data are analyzed as a function of cochlear distance/frequency, was assessed using profile analysis, with the profileR package in $R$ 3.6.1. We chose this method, because it is well suited for dealing with the between-frequency correlations in the values of any one metric in any one case (Bulut and Christopher, 2020).

\section{Results}

\section{Histopathology and audiometric thresholds}

The present study is based on quantitative histopathological analysis of 120 cases, aged 0-104 years, from the collection of archival temporal bones at the Massachusetts Eye and Ear. The 52 noise-history cases we found ranged in age from 43 to 104 years (Table 1). For a "normal" control group, we identified an age-matched cohort ( $n=51$, aged $41-93$ years) without exposure to noise, and without evidence of other explicit otologic disease. These groups were supplemented by a young normal group ( $n=17$, ages from 0 to 39 years) to allow inferences about the effects of aging per se. In each case, the fractional survival of IHCs and OHCs, ANFs and the stria vascularis was computed at numerous locations along the cochlear spiral. The histopathological data were correlated with the premortem audiometric results (i.e., pure tone audiograms and word recognition tests), which were available for 66 of the cases.

The example cases in Figure 1 illustrate the type of data obtained in each case, as well as a number of general trends. As expected, audiograms in our normal-aging cases typically showed a gradual decrease in hearing sensitivity with increasing frequency (Fig. $1 B$ ), whereas the noise history cases often showed a steeper high-frequency loss with signs of a $4-\mathrm{kHz}$ notch (Fig. $1 C)$. Hair cell losses generally increased toward the cochlear base, with near complete loss in cochlear regions where hearing levels were worse than $80-\mathrm{dB}$. OHC losses also increased with age near the apex, at cochlear regions too low in frequency to be well captured with conventional audiometric techniques. With normal aging, or in the noise-history group, ANF degeneration, which we measure here by counting the peripheral axons of these bipolar sensory neurons, was seen throughout the cochlea, but was significantly worse in the cochlear base. In contrast, strial degeneration tended to be more evenly distributed throughout the cochlear spiral.

To consider the age-related losses more quantitatively, and to parse out the effects of noise damage, we averaged histopathological metrics throughout the cochlear spiral, and plotted them against age (Fig. 2). In normal-aging ears, all the histopathological metrics decline significantly with age (Fig. $2 E$ ). The rates of decline are faster for OHCs and ANFs and relatively slower for IHCs and the stria (Fig. $2 A-D$ ). Indeed, the age-related loss of ANF peripheral axons (Fig. $2 D$ ) outstrips the rate of IHC loss (Fig. $2 A$ ) by a factor of almost $2(6.3 \%$ vs $3.4 \%$ per decade), and the slope difference is highly significant (Fig. $2 F$ ). Although the vast majority of ANFs (95\%) make synaptic contact only with IHCs, the normal age-related loss of ANFs is statistically indistinguishable from that for the OHCs rather than the IHCs (Figs. $2 C, D, F)$. The primary neural degeneration implied by these slope differences, arises mainly in apical half of the cochlea $(0.25,0.5$, and $1.0 \mathrm{kHz}$ ), where the relation between ANF and IHC survival suggests that there can be almost no surviving ANFs despite loss of only $30 \%$ of IHCs (Fig. $3 A$ ). In contrast, the IHC and ANF losses in the basal half of the cochlea $(2.0,4.0$, and $8.0 \mathrm{kHz})$ are proportional (Fig. 3C). 


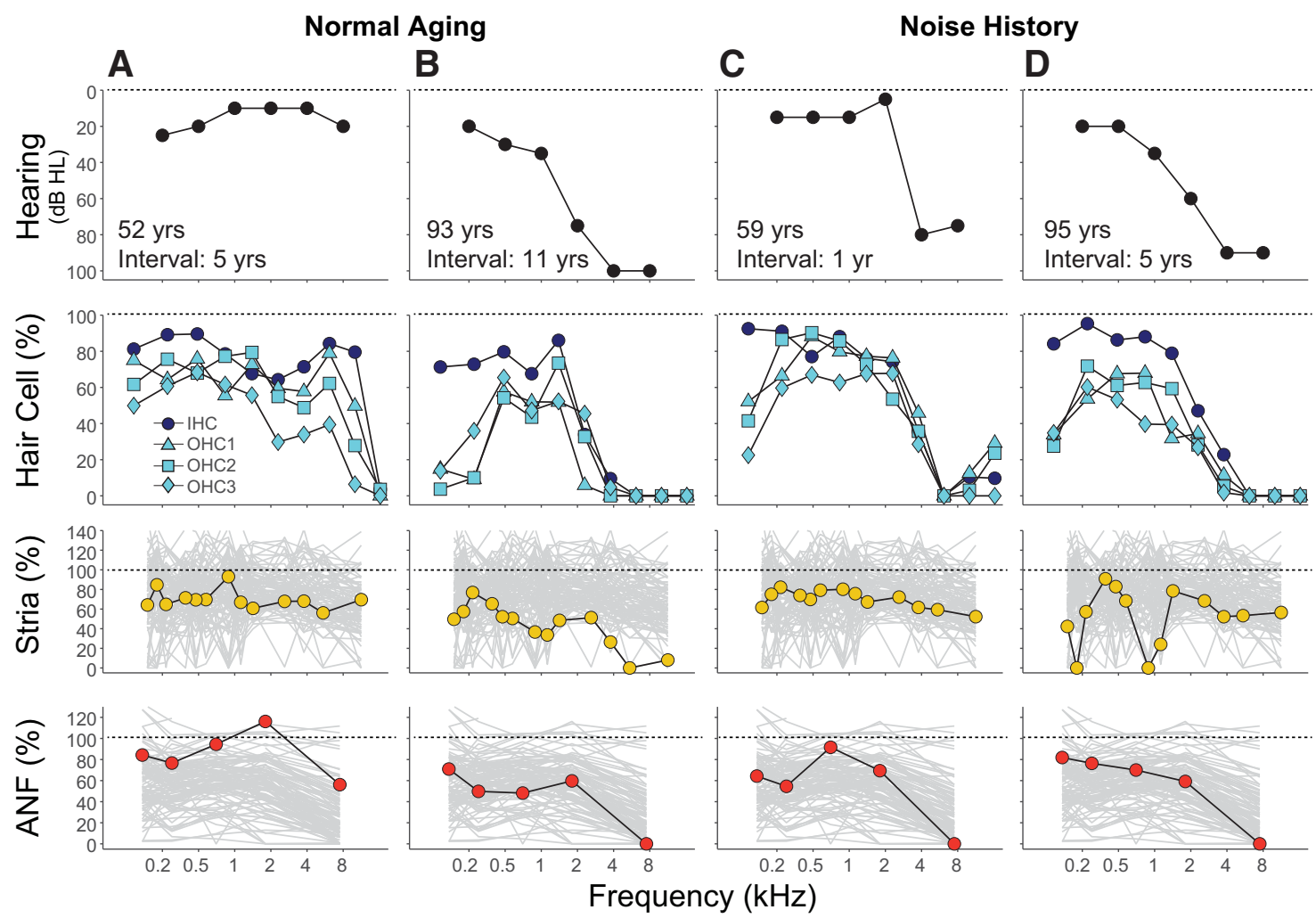

Figure 1. Histopathological analysis from four cases in the present study: two from the noise-history group $(\boldsymbol{C}, \boldsymbol{D})$ and two age-matched cases from normal-aging group $(\boldsymbol{A}, \boldsymbol{B})$. For each case, the most recent audiogram is shown in the top row, and the histopathological data for hair cells, stria vascularis, and ANFs are summarized in the second, third, and fourth rows, respectively. Hair cell data are binned into 10\% increments of cochlear length, and the key at the left applies to all columns. The first, second, and third row of $\mathrm{OHC}$ are indicated as $\mathrm{OHC1}, \mathrm{OHC2}$, and $\mathrm{OHC}$, respectively. Strial (yellow circles) and neuronal (red circles) data from each exemplar are plotted (unbinned), superimposed on the profiles from all cases (gray lines). Age and the interval between audiometric test and death are indicated on first row. For the histopathological measures, cochlear location has been converted into frequency according to the cochlear frequency map for human (Greenwood, 1990).

Since the noise-history group included no cases with age $<43$ years, the age-related rate of histologic degeneration is difficult to assess (Fig. 2). Therefore, to gauge the effects of noise, we divided the age range in half (50-74 vs 75-104 years) and compared the two groups by $t$ tests. As shown in Figure 2, the effects of noise are significant only for the 50to 74-year group, and only for the OHCs and ANFs, where noise exposure causes an additional $24 \%$ and $25 \%$ loss, respectively.

To more thoroughly investigate the effects of age and noise, we plotted histopathological metrics, separated by age and exposure history, as a function of cochlear frequency (Fig. 4). In normal aging, IHC loss is flat versus frequency in the apical half of the cochlea, increasing monotonically toward the base and worsening with age (Fig. 4A). IHC survival did not significantly differ between normal and noise-history groups when averaged across either the apical or basal halves of the cochlea for either age group (Fig. 4A,F,G).

In normal aging, both apical and basal-turn foci exhibit $\mathrm{OHC}$ loss (Fig. $4 B$ ). In the noise history group, $\mathrm{OHC}$ loss near $4 \mathrm{kHz}$ was dramatic in the 50-74 year olds, consistent with noise damage (McGill and Schuknecht, 1976). In the apical half of the cochlea, OHC losses were almost identical between normal and noise-history group, while in the basal regions, $\mathrm{OHC}$ losses were significantly greater in the 50 - to 74 -year-old noise group (Fig. $4 B, F, G ; p<0.001$ by both the slope and level tests). Correspondingly, thresholds near $4 \mathrm{kHz}$ were much worse among 50-74 year olds in the noise group, and, especially at high frequencies, the differences between noise and normal groups were highly significant (Fig. $4 E-G$; $p<0.0001$ by the slope test).

In normal aging, ANF loss was seen throughout the cochlea, although it was most severe at the basal-most measurement point. The noise-related loss of ANFs among the 50-74 year olds was relatively constant across frequency (Fig. $4 C$ ) as indicated by the profile analysis, i.e., the slopes are nearly the same, but the ANF intercepts are different (Fig. $4 F, G$ ). The difference in strial damage between the noise and normal-aging groups was insignificant in both age groups and both cochlear regions, by both slope and level tests (Fig. $4 D, F, G$ ).

Intriguingly, the noise-related differences in IHC, $\mathrm{OHC}$ and ANF survival in the older group were not statistically significant for all metrics and in both cochlear regions (Fig. $4 F, G ; p>0.05$ for both slope and level). This convergence of results as postexposure survival increases beyond an assumed retirement from workplace exposures at age 65-70 suggests that the inner ear structures affected by aging are the same as those affected by acoustic overexposure.

\section{Histopathology and word scores}

Audiological studies show that performance on word identification tests, even when the stimuli are presented at levels adjusted to insure audibility, are highly correlated with audiometric losses, especially at $0.5-2 \mathrm{kHz}$, where critical speech information is found (Halpin and Rauch, 2009). In the present study, pure-tone averages (PTAs) from 0.5 to $2 \mathrm{kHz}$ were indeed highly correlated 

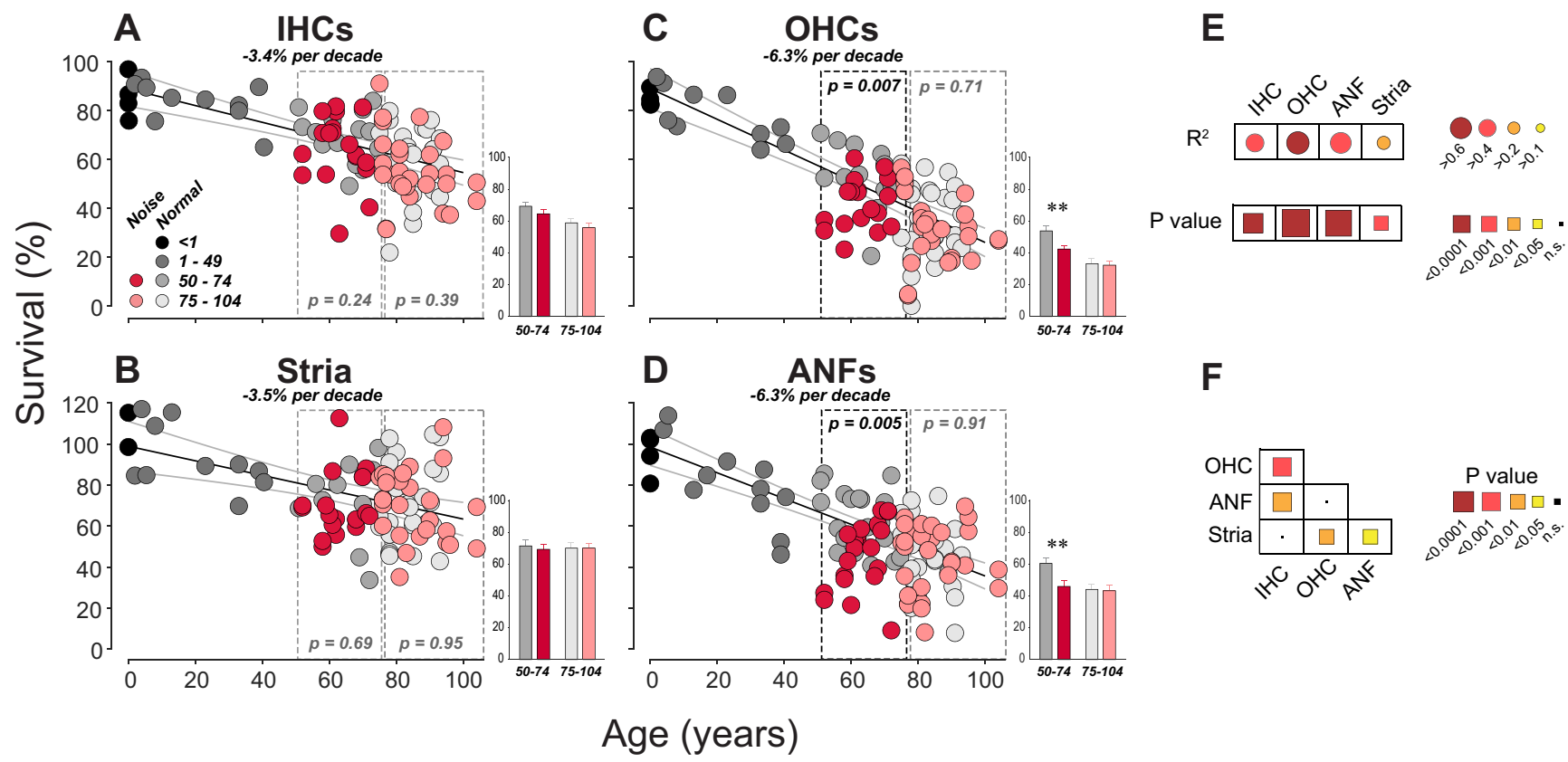

$\mathbf{F}$

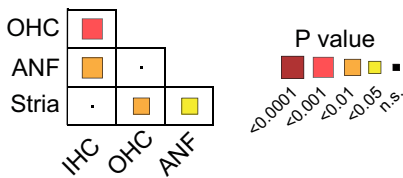

Figure 2. Age-related loss of IHCS $(A)$, stria vascularis $(B), 0 H C s(C)$, and ANF peripheral axons (D). Each point in each scatterplot represents the mean value of the corresponding metric in one case, averaged across the entire cochlear spiral. The dataset indudes all cases $(n=120)$, grouped by age, and separated into those with (red tones) or without (gray tones) a noise history (Table 1). Strial measurements were impossible in 22 cases because of gross postmortem artifact $(n=98 ; 61 \mathrm{M}, 37 \mathrm{~F})$, and ANF counts were impossible in six cases because of faint staining $(n=114 ; 73 \mathrm{M}, 41 \mathrm{~F})$, hair cell counts were impossible in 18 cases because of postmortem artifact $\left(n=102 ; 63 \mathrm{M}, 39 \mathrm{~F}\right.$ ). The regression lines (computed in MATLAB using the fittm function) and 95\% confidence intervals are derived for "normal cases," and their $R^{2}$ and $p$ value are indicated graphically in $\boldsymbol{E}$. The slope of the regression lines, i.e., rate of degeneration (per decade) is indicated beneath each panel header. The $p$ values for the significance of the pairwise slope differences (by ANCOVA: aoctool function in MATLAB) are indicated in $\boldsymbol{F}$. To compare measurements from noise-history cases to their age-matched controls, students $t$ tests were used (ttest2 in MATLAB): the resultant $p$ values are indicated in the dashed boxes, and group means ( \pm SEMs) are shown by the small bar graphs. ** indicates $p<0.01$. Key in panel $\boldsymbol{A}$ also applies to panels $\boldsymbol{B}-\boldsymbol{D}$ and their insets.
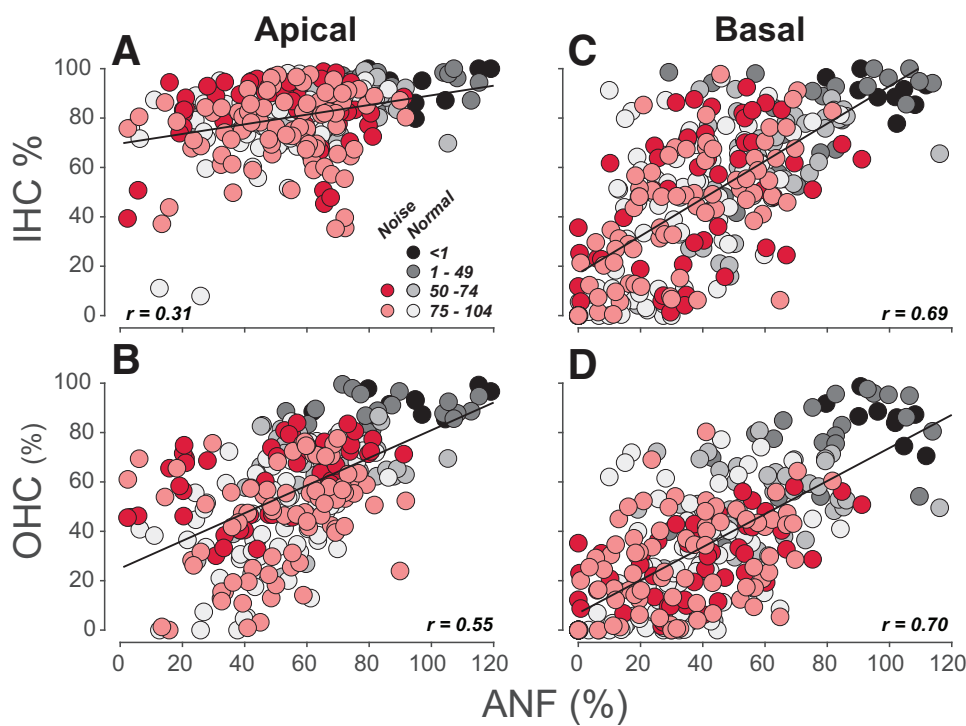

Figure 3. Pairwise correlations between the survival of ANF peripheral axons and either IHCs $(A, C)$ or $0 \mathrm{HCS}(B$, $\boldsymbol{D})$ in either the apical $(\boldsymbol{A}, \boldsymbol{B})$ or basal $(\boldsymbol{C}, \boldsymbol{D})$ cochlear regions. The histologic data in each case are binned and averaged over the cochlear locations appropriate to each audiometric frequency, thus each case produces three points in each panel: $0.25,0.5$, and $1 \mathrm{kHz}$ in apical regions; $2.0,4.0$, and $8.0 \mathrm{kHz}$ in basal regions. The leastsquare best-fit lines are shown in black, along with the Pearson's $r$ value (corrcoef in MATLAB). Data were derived from the same (96/ $120)$ cases with complete ANF and hair cell data as shown in Figure 2 ( $58 \mathrm{M}, 38 \mathrm{~F}$ ). Key in $\boldsymbol{A}$ applies to all panels.

with word score (Fig. 5A). However, there remains significant variability in performance among subjects with identical thresholds. One aim of the present study was to test the idea that, among subjects with similar PTAs, ANF survival is an important predictor of word scores.
Parsing the relative contributions of the different histopathologies to word score is complicated by the collinearity among many of the histopathological measures. As illustrated in Figure $5 B, C$, when averaged across the entire speech range (i.e., $0.5-2 \mathrm{kHz}$ ), the mean survival of IHCs, OHCs, and ANFs is each highly correlated with word score when considered individually. However, strial survival is not significantly correlated with any other histopathological or audiometric measurements.

To evaluate the relative importance of different histopathogical metrics to the prediction of word scores, we combined data from both the normal aging and the noise-history groups and conducted a multivariable regression analysis (Fig. 6). Given the fundamental differences between apical and basal cochlear regions suggested by the data in Figure 3, we divided each histologic predictor variable into a low-frequency mean and a high-frequency mean for each case. All predictors were normalized to the same scale, thus the absolute values of the LASSO coefficients (Fig. $6 B, D$ ) indicate the relative importance of each.

In one model, we considered all the histologic metrics, i.e., (1) ANF survival; (2) IHC survival; (3) OHC survival; and (4) strial survival, supplemented by (5) noise-exposure history and (6) sex. In all, 36\% of variance in word scores was explained by this model (Fig. 6A). The dominant effect of OHC survival on word score is expected, given the strong correlation between word score and thresholds 

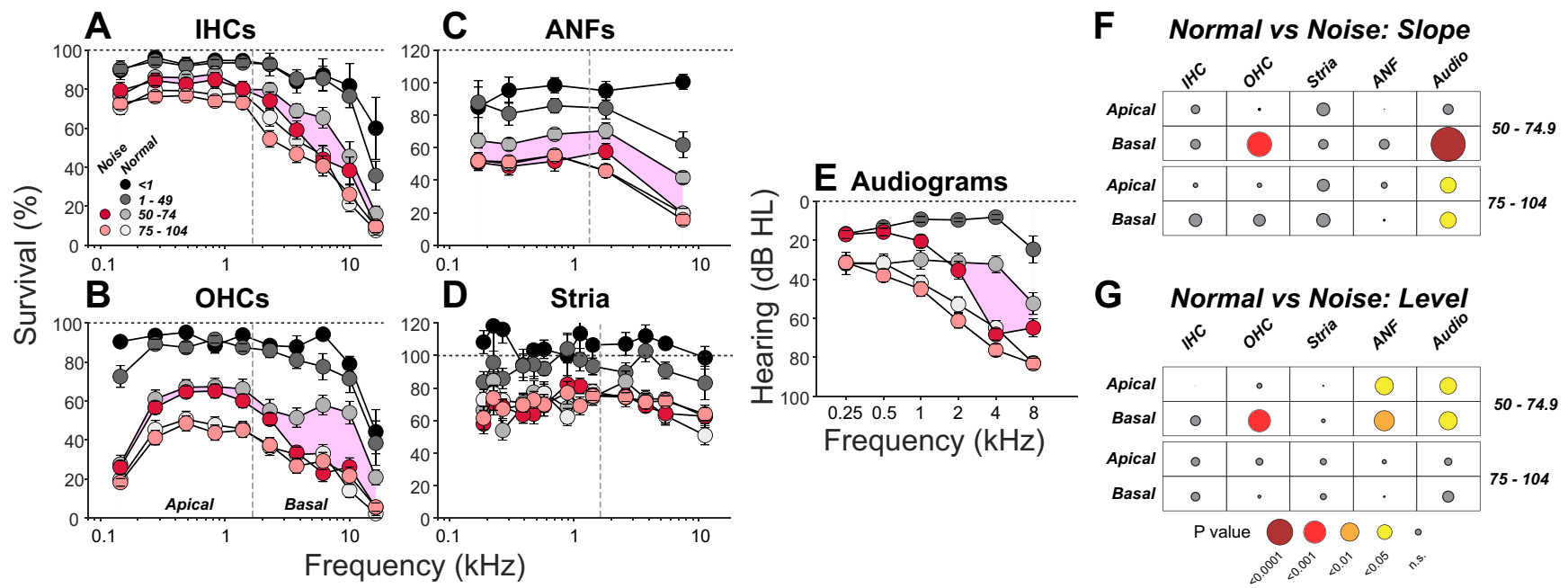

Figure 4. Histologic and audiometric differences between normal and noise-history groups as a function of cochlear location. Mean survival $( \pm$ SEMs) of IHCS $(\boldsymbol{A})$, OHCS $(\boldsymbol{B})$, ANFs $(\boldsymbol{C})$, and stria vascularis $(\boldsymbol{D})$ are grouped by age as indicated, and separated into those with or without noise-exposure history. For the histopathological measures, cochlear location has been converted into frequency according to the cochlea frequency map for human. Hair cell data are binned into $10 \%$ increments of cochlear length; strial and neuronal data are unbinned. The exposurerelated loss of IHCS, OHCS, ANFs, and hearing level in each age group are shaded to aid in visual comparison. The mean audiometric thresholds ( \pm SEMs) of the same groups are shown in $\boldsymbol{E}$. The $p$ values for differences in slope $(\boldsymbol{F})$ and level $(\boldsymbol{G})$ for noise versus normal group metrics were separately computed for low-frequency versus high-frequency regions (division line is shown in $\boldsymbol{B}$ ) and are indicated graphically by the size and color of the circles (by profile analysis: profileR package in R 3.6.1). ANF survival at $0.7 \mathrm{kHz}$ was included in profile analysis for both halves of the cochlea. Key in $\boldsymbol{A}$ applies to panels $\boldsymbol{A}-\boldsymbol{E}$, key in $\boldsymbol{G}$ applies to panels $\boldsymbol{F}, \boldsymbol{G}$.
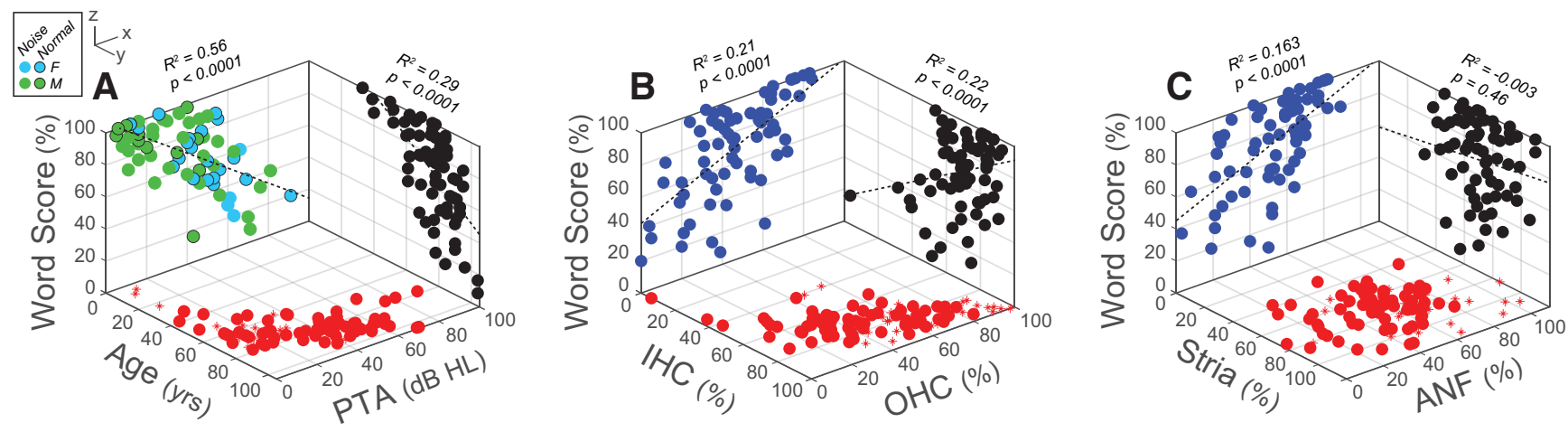

Figure 5. Pairwise comparison of word scores with age and PTA $(\boldsymbol{A})$, with $\mathrm{OHC}$ and IHC survival $(\boldsymbol{B})$ or with stria and ANF survival $(\boldsymbol{C})$. Except for age, each point in these $3 \mathrm{D}$ scatterplots shows the mean of the relevant metric for that case, averaged across cochlear loci from 0.5 to $2 \mathrm{kHz}$. The smaller symbols in the $x y$ plane indicate cases for which there are no word scores. The adjusted $R^{2}$ values and $p$ values for the least-square regression (fittm function in MATLAB) are indicated on top of each metric. Data were derived from all cases with histopathological measurements and word scores, including normal and noise-history cases, separately coded only in the zx plane of panel $\boldsymbol{A}$.

(Fig. 5A) and the dominance of $\mathrm{OHC}$ survival in predicting thresholds in these human temporal-bone specimens ( $\mathrm{Wu}$ et al., 2020a). Although the LASSO coefficients are low, ANF survival in both low and high-frequency regions contributed significantly to the performance on word-discrimination tests, as suggested by classic neurophysiological studies (Kiang and Moxon, 1974) and directly supported by recent audiological studies (Hunter et al., 2020). Presumably, the coefficients would be higher if ANF survival were not also so highly correlated with OHC and IHC survival (Fig. 3). In contrast, neither strial survival, noise history or sex significantly improved the predictive power of the model (thus no LASSO coefficients are defined), although the robustness of a conclusion re sex is limited by the small number of females in the sample.

To more specifically evaluate the contribution of ANF survival to word scores among subjects with similar hearing thresholds, PTAs and ANFs at low and high frequencies were included in the model, supplemented by noise-exposure history and sex
(Fig. 6C,D). More variance can be explained (adjusted $R^{2}=0.6$ ) when PTAs are included, and thresholds at both low and high frequencies make important contributions (Fig. 6D). In this model, the contributions of ANF survival are stronger than in the purely histologic model (Fig. $6 A, B$ ). OHC contributions are insignificant here, presumably because they are so closely tied to the PTAs (Wu et al., 2020a). Once again, strial survival is not a factor, nor are noise history or sex.

\section{Discussion}

Neural, hair cell, and strial loss in age-related hearing loss (ARHL) and noise-induced hearing loss (NIHL)

The present study was inspired by animal work showing that massive ANF degeneration can occur without IHC death after noise exposure (Kujawa and Liberman, 2009) and before IHC death in aging (Stamataki et al., 2006; Sergeyenko et al., 2013). Here, with human autopsy specimens, we corroborate prior human work showing that age-related loss of ANF peripheral 

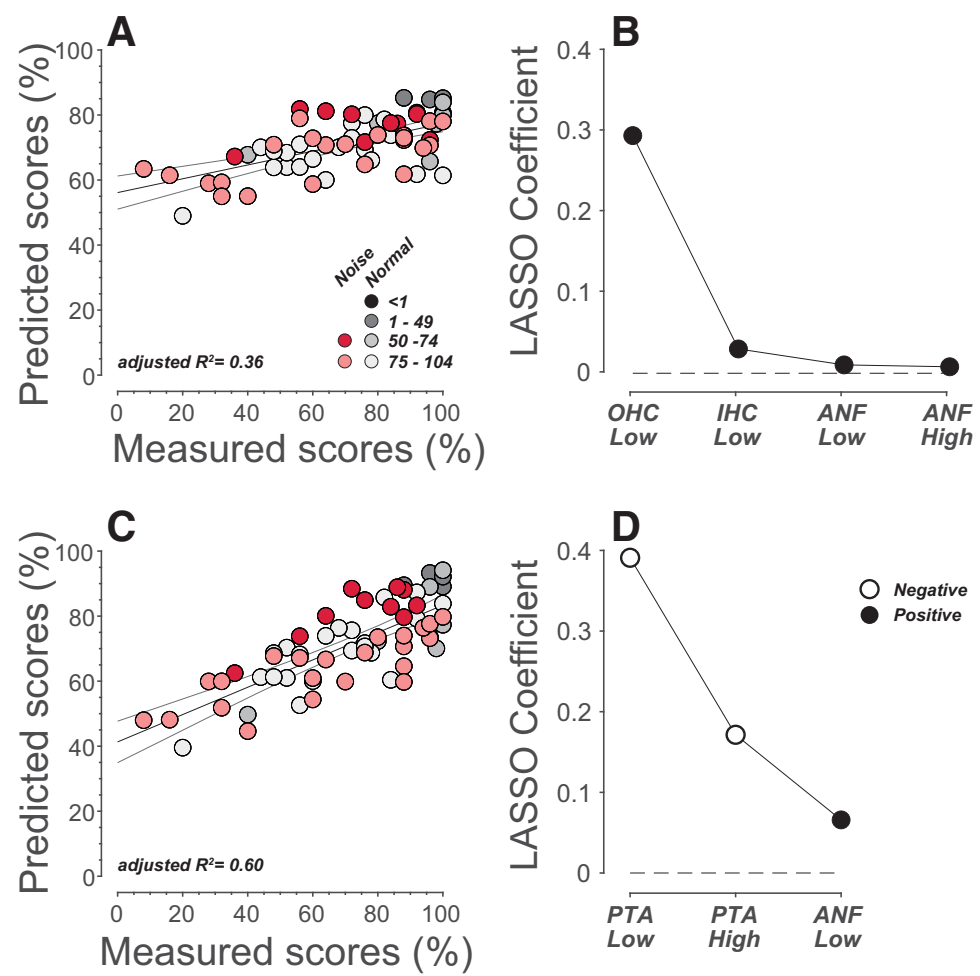

Figure 6. Multivariable LASSO regression was used to derive the relative weightings (coefficients) of histologic metrics alone $(\boldsymbol{B})$, or ANF metrics plus hearing levels $(\boldsymbol{D})$, that best predict word scores $(\boldsymbol{A}, \boldsymbol{C}$, respectively). Each metric was averaged separately for low $(<1.5 \mathrm{kHz})$ versus high $(>1.5 \mathrm{kHz})$ frequencies, noise exposure history and sex were included in both models. Coefficients are shown only for those metrics that improved the model prediction. Measured versus predicted word scores $(\boldsymbol{A}, \boldsymbol{C})$ are shown with the least-square regression line and $95 \%$ confidence intervals. Data were derived from the 66 cases with recent word test, audiogram (60/66 case were tested $<6$ years before death), and complete histologic data. Cases are grouped by age, and noise history as shown in the key. The differences in coefficient polarity $(\boldsymbol{B}, \boldsymbol{D})$ arise because word scores are positively correlated with histologic metrics (\% survival) and negatively correlated with PTA.

axons outstrips IHC loss by roughly a factor of 2 (Wu et al., 2018). Present data further suggests that noise-exposure accelerates ANF loss in all cochlear regions, with minimal effect on IHC survival (Figs. 2, 3). Thus, as in animal models, primary ANF degeneration in human NIHL leaves surviving IHCs partially deafferented, and noise exposure indeed exacerbates primary neural degeneration in humans.

Our data also show a positive correlation between $\mathrm{OHC}$ loss and ANF loss throughout the cochlea (Fig. $3 B, D$ ), which is unexpected since $95 \%$ of ANFs make synaptic contact only with IHCs (Liberman, 1982). In contrast, animal studies report a negative correlation between OHC and ANF synaptic loss (Fernandez et al., 2020), suggesting that OHC loss reduces neuropathy by reducing the sound-evoked drive to the IHC synapse. The discrepancy may arise because such "protection" only occurs during the brief, high-level exposures used in animal experiments, which render affected cochlear regions unresponsive to sound during the exposure, as evidenced by the large threshold shifts seen immediately postexposure (Fernandez et al., 2020). In humans with workplace noise exposure, acute thresholds shifts at the end of each workday are minimal, and the accumulation of permanent NIHL is gradual (Passchier-Vermeer, 1974). Thus, the damage mechanisms underlying cell death in animal studies are likely different from those caused by the multiple, long-duration, moderate-level exposures of human occupational noise.

The correlations between OHC loss and neural loss are a critical clue in the ongoing search for "hidden hearing loss" in humans. The animal studies showing that noise exposure can destroy ANF/IHC synapses without causing permanent threshold elevation or hair cell loss (Kujawa and Liberman, 2009) resurrected the old idea that cochlear neural loss has minimal effect on thresholds and thus "hides" behind the audiogram (Schuknecht and Woellner, 1955). In the animal work, the suprathreshold amplitude of auditory evoked potentials could be diagnostic of this synaptopathy (Kujawa and Liberman, 2009; Sergeyenko et al., 2013), but only if cochlear thresholds had returned to normal. It inspired a host of human studies comparing noise history, speech discrimination and/or suprathreshold amplitudes of auditory-evoked neural potentials in subjects with normal audiograms (Liberman et al., 2016; Bramhall et al., 2019; Prendergast et al., 2019). Present data suggest that noise-induced degeneration of ANF peripheral axons in humans may not be sizable without sizable $\mathrm{OHC}$ losses, which will also elevate audiometric thresholds. Thus, neural degeneration may "hide" behind many human audiograms, but it may not be as disabling, or as common, in "normal-hearing" subjects as it is in those with threshold elevation.

A similar discrepancy between animal and human studies is seen in the patterns of strial atrophy. Animal studies show that the stria can be permanently damaged by high-level noise (Hirose and Liberman, 2003), but only in cases where the entire organ of Corti has degenerated into an undifferentiated epithelium (Liberman and Kiang, 1978; Liberman and Mulroy, 1982; Wang et al., 2002; Hirose and Liberman, 2003). Here, there were no significant differences in strial survival between normal and noise groups (Fig. 4). This discrepancy, again, may reflect the fact that occupational exposures in humans are not as acutely catastrophic as the high-intensity, short-duration exposures in animal studies. Indeed, human cases of occupational noise damage do not show the mid-frequency wipe-outs of the sensory epithelium (Bredberg, 1968; McGill and Schuknecht, 1976) that characterize the animal experiments in which permanent strial damage is observed.

\section{Hair cell loss and threshold shifts in ARHL versus NIHL}

Animal studies of NIHL show that the key structural changes underlying the permanent threshold shifts are (1) loss of OHCs, the "cochlear amplifiers" whose electromotile properties enhance the mechanical vibrations of the sensory epithelium; and (2) damage to the stereocilia bundles on both IHCs and OHCs, where the mechanoelectric transduction channels are located (Liberman and Dodds, 1984). Focal loss of IHCs can lead to "dead zones," but IHCs are less susceptible to noise (Liberman and Kiang, 1978). Furthermore, scattered IHC loss, per se, does not significantly affect thresholds until it reaches $80 \%$ (Lobarinas et al., 2013), and subtotal ANF loss of 50-80\% also has little effect on thresholds for tones in quiet (Schuknecht and Woellner, 1955).

In humans, noise exposure leads to a "notched" audiogram with the worst threshold shifts at $4 \mathrm{kHz}$ (Passchier-Vermeer, 
1974; McBride and Williams, 2001). Since there are no other etiologies known to produce this audiometric pattern (McBride and Williams, 2001), the $4 \mathrm{kHz}$ notch in our "noise" population (Fig. 4E), and its absence in the age-matched non-noise group, provides evidence that our sample grouping reflects important differences in lifetime noise exposure. Correspondingly, OHC loss in our NIHL group also differ most dramatically from control near 4-kHz, at least in the 50- to 74-year-old group (Fig. 4B).

As the years of exposure increase, the $4 \mathrm{kHz}$ notch among workers in noisy factories spreads toward higher frequencies (Passchier-Vermeer, 1974), because the base of the cochlea is more vulnerable to a wide range of cochlear insults. Here, with increasing age, the noise and control groups also showed increasing threshold shifts at high frequencies, but surprisingly they converged with respect to audiometric threshold and cochlear histopathology after age 75 (Fig. 4). This unexpected finding suggests that the vulnerabilities of cochlear structures to aging versus occupational noise are similar, if extended to the end of the human lifespan. Perhaps much of "normal" cochlear aging in humans represents a slow accumulation of avoidable ear abuse, as suggested by classic studies of ARHL in non-industrialized societies, where people can age with minimal high-frequency threshold shift (Rosen et al., 1962).

In animal studies of cochlear aging, conducted in a highly controlled acoustic environment, there is an apical focus of OHC loss as well as strial degeneration throughout the cochlea (Tarnowski et al., 1991), similar to that seen here, but little OHC loss in the basal half of the cochlea compared with our aging humans: compare Tarnowski et al., (1991; their Fig. 7) to Figure 4 here. The fact that our human data shows no effect of noise history on apical OHC loss, or the pan-cochlear strial degeneration (Fig. 4), suggests that these are purely age-related effects, while much of the basal OHC loss, and the high-frequency hearing loss it produces, is apparently exacerbated by noise and may be an avoidable consequence of lifetime ear abuse.

\section{Neural degeneration and word scores}

As predicted from animal work (Schuknecht and Woellner, 1955), human studies suggest that the audiogram is best predicted by OHC and IHC survival, with minimal contribution of ANF degeneration (Wu et al., 2020a). Nevertheless, the loss of ANF information channels likely degrades encoding of complex acoustic stimuli in central auditory pathways (Chambers et al., 2016; Lobarinas et al., 2016). Indeed, human studies have suggested that loss of ANFs (Felder and Schrott-Fischer, 1995) or spiral ganglion cells (Otte et al., 1978) is associated with poor word-recognition scores. However, prior studies have included small numbers of cases and did not account for the confound of threshold elevation, which is strongly correlated with word scores (Fig. 5A).

Here, we control for the contribution of threshold shift, and the collinearity of many histopathological metrics (Fig. 5), through a multivariable LASSO regression model. Our hypothesis was that ANF survival would be predictive of word score even when PTA is included in the regression model. Indeed, when included as an input variable along with the histopathological metrics, PTA is the most important predictor of word scores (Fig. 6D), but ANF survival is the only other variable significantly associated with the outcome (Fig. 6D). Looking at the data another way, the fact that threshold (i.e., PTA) is a strong predictor of word score even when test words are presented at a clearly audible level ( $30 \mathrm{~dB}$ above threshold) may arise because, as OHC losses increase (and therefore also PTAs), so do the (otherwise hidden) ANF losses (Fig. 3A). Thus, the neural loss, although not reflected in audiometric thresholds, may be the main driver of the observed reduction in discriminability of speech seen as PTA increases (Fig. 6D).

In many ways, ANF loss, or synaptic disconnection, should be functionally similar to IHC loss, as both reduce the number of independent channels sending information to the central auditory processor. Both should be fundamentally different from OHC loss, which affects tuning, cochlear mechanical nonlinearities and the strength of the cochlear amplifier. On the other hand, subtotal loss of ANFs differs from subtotal loss of IHCs in that the former may preferentially affect ANFs with high thresholds and low spontaneous rates (SRs; Furman et al., 2013), whereas the latter will simultaneously silence equal proportions of ANFs from all SR groups. Low-SR fibers, by virtue of their high thresholds, are particularly important in noisy backgrounds (Costalupes et al., 1984), thus, the key contributors to the predictive models might have been different if tied to words-in-noise tests rather than words delivered in quiet.

Given preclinical progress in gene therapy for hereditary hearing loss (Nist-Lund et al., 2019), it is important to know the minimal number of IHCs required to provide useful word recognition. The data in Figure $5 B$ show that the probability of good speech discrimination grows significantly when IHC survival exceeds 55\%, although more data are needed on this important question. It is also important for clinical trials of cochlear regenerative therapies to know which etiologies of hearing impairment include significant neural and/or strial degeneration, which might "hide" behind the audiogram. Here, we show that although noise damage is unlikely to produce additional strial damage, it is likely to add to the level of cochlear neural degeneration because of aging alone.

\section{References}

Arenas JP, Suter AH (2014) Comparison of occupational noise legislation in the Americas: an overview and analysis. Noise Health 16:306-319.

Bramhall N, Beach EF, Epp B, Le Prell CG, Lopez-Poveda EA, Plack CJ, Schaette R, Verhulst S, Canlon B (2019) The search for noise-induced cochlear synaptopathy in humans: mission impossible? Hear Res 377:88103.

Bredberg G (1968) Cellular pattern and nerve supply of the human organ of Corti. Acta Otolaryngol Suppl 236:1+.

Bulut O, Christopher DD (2020) Profile analysis of multivariate data: a brief introduction to the profiler package. PsyArXiv. doi: 10.31234/osf.io/sgy8m.

Chambers AR, Resnik J, Yuan Y, Whitton JP, Edge AS, Liberman MC, Polley DB (2016) Central gain restores auditory processing following near-complete cochlear denervation. Neuron 89:867-879.

Chen MA, Webster P, Linthicum YE, Jr FH (2006) Presbycusic neuritic degeneration within the osseous spiral lamina. Otol Neurotol 27:316322 .

Costalupes JA, Young ED, Gibson DJ (1984) Effects of continuous noise backgrounds on rate response of auditory nerve fibers in cat. J Neurophysiol 51:1326-1344.

Felder E, Schrott-Fischer A (1995) Quantitative evaluation of myelinated nerve fibres and hair cells in cochleae of humans with age-related hightone hearing loss. Hear Res 91:19-32.

Fernandez KA, Guo D, Micucci S, De Gruttola V, Liberman MC, Kujawa SG (2020) Noise-induced cochlear synaptopathy with and without sensory cell loss. Neuroscience 427:43-57.

Furman AC, Kujawa SG, Liberman MC (2013) Noise-induced cochlear neuropathy is selective for fibers with low spontaneous rates. J Neurophysiol 110:577-586.

Gordon-Salant S (2005) Hearing loss and aging: new research findings and clinical implications. J Rehabil Res Dev 42:9-24.

Greenwood DD (1990) A cochlear frequency-position function for several species-29 years later. J Acoust Soc Am 87:2592-2605. 
Halpin C, Rauch SD (2009) Clinical implications of a damaged cochlea: pure tone thresholds vs information-carrying capacity. Otolaryngol Head Neck Surg 140:473-476.

Hirose K, Liberman MC (2003) Lateral wall histopathology and endocochlear potential in the noise-damaged mouse cochlea. J Assoc Res Otolaryngol 4:339-352.

Hunter LL, Monson BB, Moore DR, Dhar S, Wright BA, Munro KJ, Zadeh LM, Blankenship CM, Stiepan SM, Siegel JH (2020) Extended high frequency hearing and speech perception implications in adults and children. Hear Res 397:107922.

Kiang NY, Moxon EC (1974) Tails of tuning curves of auditory-nerve fibers. J Acoust Soc Am 55:620-630.

Kujawa SG, Liberman MC (2009) Adding insult to injury: cochlear nerve degeneration after "temporary" noise-induced hearing loss. J Neurosci 29:14077-14085.

Kujawa SG, Liberman MC (2019) Translating animal models to human therapeutics in noise-induced and age-related hearing loss. Hear Res 377:4452 .

Liberman MC (1982) Single-neuron labeling in the cat auditory nerve. Science 216:1239-1241.

Liberman MC, Kiang NY (1978) Acoustic trauma in cats. Cochlear pathology and auditory-nerve activity. Acta Otolaryngol Suppl 358:1-63.

Liberman MC, Mulroy MJ (1982) Acute and chronic effects of acoustic trauma: cochlear pathology and auditory nerve pathophysiology. In: New perspectives on noise-induced hearing loss (Hamernik RP, Henderson D, Salvi R, eds), pp 105-136. New York: Raven Press.

Liberman MC, Dodds LW (1984) Single-neuron labeling and chronic cochlear pathology. III. Stereocilia damage and alterations of threshold tuning curves. Hear Res 16:55-74.

Liberman MC, Epstein MJ, Cleveland SS, Wang H, Maison SF (2016) Toward a differential diagnosis of hidden hearing loss in humans. PLoS One 11:e0162726.

Liu W, Edin F, Atturo F, Rieger G, Löwenheim H, Senn P, Blumer M, Schrott-Fischer A, Rask-Andersen H, Glueckert R (2015) The pre- and post-somatic segments of the human type I spiral ganglion neuronsstructural and functional considerations related to cochlear implantation. Neuroscience 284:470-482.

Lobarinas E, Salvi R, Ding D (2013) Insensitivity of the audiogram to carboplatin induced inner hair cell loss in chinchillas. Hear Res 302:113-120.

Lobarinas E, Salvi R, Ding D (2016) Selective inner hair cell dysfunction in chinchillas impairs hearing-in-noise in the absence of outer hair cell loss. J Assoc Res Otolaryngol 17:89-101.

McBride DI, Williams S (2001) Audiometric notch as a sign of noise induced hearing loss. Occup Environ Med 58:46-51.

McGill TJ, Schuknecht HF (1976) Human cochlear changes in noise induced hearing loss. Laryngoscope 86:1293-1302.

Merchant SN, Nadol JB (2010) Schuknecht's pathology of the ear, Ed 3. Shelton: People's Medical Publishing House.
Merchant SN, McKenna MJ, Adams JC, Nadol JB Jr, Fayad J, Gellibolian R, Linthicum FH Jr, Ishiyama A, Lopez I, Ishiyama G, Baloh R, Platt C (2008) Human temporal bone consortium for research resource enhancement. J Assoc Res Otolaryngol 9:1-4.

Nist-Lund CA, Pan B, Patterson A, Asai Y, Chen T, Zhou W, Zhu H, Romero S, Resnik J, Polley DB, Géléoc GS, Holt JR (2019) Improved $\mathrm{TMC} 1$ gene therapy restores hearing and balance in mice with genetic inner ear disorders. Nat Commun 10:236

O’Malley JT, Burgess BJ, Jones DD, Adams JC, Merchant SN (2009) Techniques of celloidin removal from temporal bone sections. Ann Otol Rhinol Laryngol 118:435-441.

Otte J, Schunknecht HF, Kerr AG (1978) Ganglion cell populations in normal and pathological human cochleae. Implications for cochlear implantation. Laryngoscope 88:1231-1246.

Passchier-Vermeer W (1974) Hearing loss due to continuous exposure to steady-state broad-band noise. J Acoust Soc Am 56:1585-1593.

Prendergast G, Guest H, Munro KJ, Kluk K, Léger A, Hall DA, Heinz MG, Plack CJ (2017) Effects of noise exposure on young adults with normal audiograms I: electrophysiology. Hear Res 344:68-81

Prendergast G, Couth S, Millman RE, Guest H, Kluk K, Munro KJ, Plack CJ (2019) Effects of age and noise exposure on proxy measures of cochlear synaptopathy. Trends Hear 23:2331216519877301.

Rosen S, Bergman M, Plester D, El-Mofty A, Satti MH (1962) Presbycusis study of a relatively noise-free population in the Sudan. Ann Otol Rhinol Laryngol 71:727-743.

Schuknecht HF, Woellner RC (1955) An experimental and clinical study of deafness from lesions of the cochlear nerve. J Laryngol Otol 69:75-97.

Sergeyenko Y, Lall K, Liberman MC, Kujawa SG (2013) Age-related cochlear synaptopathy: an early-onset contributor to auditory functional decline. J Neurosci 33:13686-13694.

Stamataki S, Francis HW, Lehar M, May BJ, Ryugo DK (2006) Synaptic alterations at inner hair cells precede spiral ganglion cell loss in aging C57BL/6J mice. Hear Res 221:104-118.

Tarnowski BI, Schmiedt RA, Hellstrom LI, Lee FS, Adams JC (1991) Agerelated changes in cochleas of mongolian gerbils. Hear Res 54:123-134.

Wang Y, Hirose K, Liberman MC (2002) Dynamics of noise-induced cellular injury and repair in the mouse cochlea. J Assoc Res Otolaryngol 3:248268.

Wu PZ, Liberman LD, Bennett K, de Gruttola V, O’Malley JT, Liberman MC (2018) Primary neural degeneration in the human cochlea: evidence for hidden hearing loss in the aging ear. Neuroscience 407:8-20.

Wu PZ, O'Malley JT, de Gruttola V, Liberman MC (2020a) Age-related hearing loss is dominated by damage to inner ear sensory cells, not the cellular battery that powers them. J Neurosci 40:6357-6366.

Wu PZ, Wen WP, O'Malley JT, Liberman MC (2020b) Assessing fractional hair cell survival in archival human temporal bones. Laryngoscope 130:487-495. 